\section{Das Mobilfunk-Risiko aus ärztlicher Sicht}

\author{
von Andreas D. Kappos, \\ Bundesärztekammer ${ }^{1}$
}

\begin{abstract}
Auch die Ergebnisse des Deutschen Mobilfunk-Forschungsprogramms räumen nicht alle Bedenken bezüglich der gesundheitlichen Unbedenklichkeit elektromagnetischer Felder aus. Dies betrifft insbesondere die Langzeitwirkungen, deren Bedeutung für die Gesundheit des Menschen aus methodischen Gründen zurzeit epidemiologisch nicht abzuklären ist. Aus präventivmedizinischer Sicht ist deshalb eine Minimierung der Exposition zu fordern. Das dabei zu beachtende Risikokommunikationsproblem wird aufgezeigt. Klinisch ist die Frage einer Existenz des Phänomens „Elektrosensibilität" bisher ungelöst. Dies wird anhand eines Beispiels aufgezeigt. Unbestritten ist, dass in der klinischen Praxis tätige Ärzte mit schwer leidenden Patienten konfrontiert werden, die ihre Beschwerden auf die Exposition mit EMF zurückführen. Ärztliche Aufgabe ist es, diesen Patienten die bestmögliche Hilfe zu gewähren.
\end{abstract}

\section{Einleitung}

Ärzte beschäftigen sich seit mehr als einem Jahrhundert mit den gesundheitlichen Wirkungen elektromagnetischer Strahlung auf menschliches Gewebe. Ausgangspunkt waren aber nicht die „schädlichen“, sondern die therapeutischen Wirkungen, die 1909 besonders durch Karl Franz Nagelschmidt unter dem Namen Diathermie in die Medizin eingeführt wurden (Nagelschmidt 1909). Dabei wurde die Eigenschaft hochfrequenter elektromagnetischer Strahlung genutzt, um in der Tiefe des Organismus liegendes Gewebe selektiv zu erwärmen. Auch heute noch werden in der Naturheilkunde und der physikalischen Therapie Bestrahlungen mit Kurzwellen (27,12 $\mathrm{MHz}$ ), Dezimeterwellen (434 MHz) und Mikrowellen $(2,45 \mathrm{GHz})$ eingesetzt. Dabei kommen Leistungen von mehreren hundert Watt zur Anwendung.
Die der medizinischen Anwendung zugrunde liegenden biophysikalischen Wirkungsmechanismen wurden in den $30 \mathrm{er}$ und 40er Jahren des letzten Jahrhunderts am Kaiser-Wilhelm-Institut (dem späteren MaxPlanck-Institut) für Biophysik in Frankfurt a. M. unter anderen von Herman Paul Schwan und Mitarbeitern erforscht (Schwan, Piersol 1954; Schwan, Piersol 1955; Schwan 1957). Bereits zu dieser Zeit bestand eine heftige wissenschaftliche Kontroverse, ob neben den eindeutig nachweisbaren thermischen Wirkungen auch ,athermische Wirkungen“" auftreten und gegebenenfalls bei einer Risikobewertung berücksichtigt werden müssen. Die überwiegende wissenschaftliche Meinung war und ist eigentlich bis heute, dass schädliche Wirkungen, die z. B. zur Ableitung von Grenzwerten zum Schutze der Gesundheit der Bevölkerung herangezogen werden müssen, ausschließlich auf thermische Effekte zurückgeführt werden können. Im Niedrigdosisbereich, d. h. unterhalb der thermisch definierten Grenzwerte, sollen athermische Effekte, wenn sie denn überhaupt existieren, keine gesundheitliche Relevanz haben.

Als athermische Wirkungen wären bei hochfrequenten elektromagnetischen Feldern Einflüsse zu betrachten, die ohne Erwärmung des Gewebes effektiv sind. Über solche Wirkungen ist häufig in der Literatur berichtet worden. ${ }^{2}$ Dabei handelte es sich beispielsweise um Befindlichkeitsstörungen, Beeinflussungen der kognitiven Leistungen, Schlafstörungen, Veränderungen des Elektroenzephalogramms (EEG), Induktion oder Promotion von Tumorerkrankungen, Beeinflussung des intrazellulären Kalziumhaushalts oder Veränderungen der Blut-Hirn-Schranke, gentoxische oder reproduktionstoxische Effekte. Die wissenschaftlichen Publikationen, in denen über solche Befunde berichtet wurde, sind meist Einzelarbeiten. In der Regel fehlt die Reproduktion dieser Befunde durch Arbeiten anderer Forschergruppen. Sie können also nicht als wissenschaftlich gesichert angesehen werden. Man kann sie bestenfalls als Hinweise für gesundheitsschädliche Risiken ansehen, die einer weiteren Überprüfung bedürfen. 


\section{Würdigung der Ergebnisse des DMF}

Im Rahmen des Deutschen Mobilfunk-Forschungsprogramms (DMF) wurde viel Mühe darauf verwandt, einen Teil der Lücken im wissenschaftlichen Kenntnisstand zu den athermischen Wirkungen im Niedrigdosisbereich zu schließen. In keinem der durchgeführten Forschungsprojekte konnte der Nachweis einer athermischen Wirkung geführt werden. Wie auch die bewertende Stellungnahme der Strahlenschutzkommission aufführt, bestehen allerdings nach wie vor Wissenslücken und damit weiterer Forschungsbedarf. Insbesondere die Frage nach möglichen athermischen Wechselwirkungsmodellen, die Frage nach individuell spezifischer Elektrosensibilität bestimmter Personen oder Personengruppen und die Möglichkeit von Langzeitwirkungen ist weitgehend ungeklärt. Epidemiologische Untersuchungen, die zur Klärung z. B. der Frage der Kanzerogenität elektromagnetischer Felder aus dem Mobilfunk beim Menschen beitragen könnten, stoßen auf bisher unüberbrückbare methodische Schwierigkeiten; dies ist insbesondere bei der Bestimmung der individuellen Exposition der Fall.

Die Ergebnisse des DMF können somit nicht als Beleg für die Nichtexistenz athermischer Wirkungen der elektromagnetischen Felder interpretiert werden. Seit der Beobachtung des sogenannten „Radiowellen-Syndroms" durch Erwin Schliephake 1932 (Schliephake 1932) wurde, wie oben angedeutet, eine große Zahl von Arbeiten publiziert, die funktionelle Gesundheitsstörungen im Zusammenhang mit der Exposition durch hochfrequente elektromagnetische Strahlung beschreiben. Ebenso existieren plausible pathophysiologische Erklärungsmodelle nichtthermischer Wirkungen auf die komplexen Regulationsmechanismen des menschlichen Organismus. Diese sind nicht ohne Weiteres von der Hand zu weisen. Eine umfangreiche Zusammenstellung aus alternativmedizinischer Sicht findet sich z. B. bei Hecht (2008).

Für die Ärzteschaft ergeben sich für den Umgang mit eventuellen gesundheitlichen Risiken aus der massenhaften Verbreitung des Mobilfunks und der daraus resultierenden exponentiellen Zunahme der Exposition der gesamten Bevölkerung mit elektromagnetischer Strahlung die Aspekte „Prävention“ und
„Elektrosensibilität“, die besondere Bedeutung besitzen. Diese werden im Folgenden aufgegriffen.

\section{Der präventionsmedizinische Aspekt}

Der erste Aspekt ist der der Prävention. Er betrifft die ärztliche Verpflichtung, Leben und Gesundheit des Menschen zu schützen und eine hohe Lebensqualität zu gewährleisten. In Anbetracht der vielen noch offenen wissenschaftlichen Fragen bezüglich der gesundheitlichen Bedeutung einer möglicherweise lebenslangen Exposition mit hochfrequenten elektromagnetischen Strahlen kann der Arzt nur zur Vorsicht mahnen. Ärztlicherseits ist zu fordern: Bevor die Unbedenklichkeit der lebenslangen Exposition nicht evident ist, sind alle technischen und organisatorischen Möglichkeiten zu nutzen, um die Emission elektromagnetischer Strahlen im Umfeld des Menschen zu minimieren. Mit anderen Worten ist $\mathrm{zu}$ fordern, dass Mobil- und DEC-Telefone sowie drahtlose Computereinrichtungen möglichst strahlungsarm arbeiten und mit Vorrichtungen versehen sind, die sie automatisch abschalten, wenn sie nicht in Gebrauch sind. Die Emissionen der einzelnen Geräte und die daraus resultierenden Risiken müssen für den Verbraucher nachvollziehbar sein. Somit ergeben sich die Forderungen nach einer verständlichen Kennzeichnung der verbrauchernahen Geräte und nach unparteiischer Aufklärung. Der Nutzer von Geräten, die elektromagnetische Strahlung emittieren, muss darüber aufgeklärt werden, wie er bei der Verwendung dieser Geräte sein gesundheitliches Risiko gering halten kann. Darüber hinaus müssen individuelle Verhaltensweisen aufgezeigt werden, die die Exposition des Nutzers und seiner Umgebung minimieren (z. B. durch die Verwendung von Freisprechanlagen um einen größeren Abstand zwischen dem Kopf und dem Mobiltelefon einzuhalten oder durch die Einschränkung der Gesprächsdauer).

Kinder und Jugendliche sind besonders zu schützen. Das sich noch entwickelnde kindliche Nervensystem ist besonders empfindlich gegenüber den verschiedensten Noxen (von Mühlendahl, Otto 2008) und somit vermutlich auch gegenüber hochfrequenten 
elektromagnetischen Feldern, soweit sie auf das Nervensystem wirken. Kinder und Jugendliche stehen am Anfang ihres Lebens. Die Exposition währt möglicherweise noch viele Jahrzehnte. Gerade langfristigen Wechselwirkungen hochfrequenter elektromagnetischer Felder mit dem menschlichen Organismus sind noch weitgehend ungeklärt und vermutlich auch nicht in unmittelbarer Zukunft zu erforschen, da sich die Expositionsbedingungen ständig verändern. Dies betrifft sowohl den technisch verwendeten Frequenzbereich als auch die Leistungsdichte der Exposition mit unterschiedlichen Frequenzen, die durch die verschiedensten, von den Anbietern intensiv beworbenen Anwendungen, massiv zugenommen hat. Aus ärztlicher Sicht ist deshalb die Empfehlung der Strahlenschutzkommission zu begrüßen. Sie empfiehlt, dass „Eltern, insbesondere bei Kindern im Vorschulalter, über die Nutzung von Mobiltelefonen unter Abwägung des Nutzens verantwortungsbewusst und umso restriktiver entscheiden, je jünger ein Kind ist" (SSK 2006).

Ein Risikokommunikationsproblem zwischen Arzt und Patient ergibt sich aus dem Sachverhalt, dass zwar bisher keine schädlichen Wirkungen hochfrequenter elektromagnetischer Strahlung wissenschaftlich belegt sind, dass aber gleichzeitig zu vorsichtigem Verhalten beim Umgang mit entsprechenden Geräten geraten werden muss. Es ist schwer vermittelbar, dass nicht überprüfte Hinweise auf die Möglichkeit einer schädlichen Wirkung keine Gefahr in dem Sinne bedeuten, dass ordnungsrechtliche Maßnahmen (z. B. zum Entzug der Genehmigung einer Mobilfunk-Antenne) ergriffen werden müssen. Sie sind vielmehr lediglich Anlass zu freiwilligem, individuellem, vorsorglichem Verhalten mit dem Ziel, alle denkbaren Risiken für die Betroffenen einzugrenzen. Der einzelne Arzt wird sich zur Bewältigung dieses kommunikativen Dilemmas eher auf seine subjektive Werthaltung stützen müssen als auf klare wissenschaftliche Erkenntnisse. Je nach persönlicher Einstellung wird er mehr die „Unschädlichkeit“ der elektromagnetischen Strahlen oder die „Notwendigkeit" zur Vorsorge bei fehlendem Wissen hervorheben. In jedem Fall ist es eine Wanderung auf einem schmalen Grat zwischen Sorglosigkeit und Angstmache.

\section{Das Phänomen der Elektrosensibilität}

Der zweite Aspekt der ärztlichen Beschäftigung mit gesundheitlichen Wirkungen elektromagnetischer Strahlung ist der klinischkurative. Er betrifft die Verpflichtung des Arztes, Kranken ihr Leiden zu mildern und nach bestem Wissen alles zu tun, um Gesundheit und Lebensqualität wiederherzustellen. Wie ist aber mit Personen umzugehen, die angeben, unter Einwirkung hochfrequenter elektromagnetischer Felder zu leiden und die über die unterschiedlichsten Beeinträchtigungen und Gesundheitsstörungen klagen, wenn sie entsprechend exponiert sind?

Dieser Aspekt ist eng verknüpft mit dem Phänomen der „Elektrosensibilität“". Abgesehen vom Auge, das nur auf das Spektrum des sichtbaren Lichtes reagiert, fehlt dem Menschen ein besonderes Organ zur Wahrnehmung elektromagnetischer Strahlung. Trotzdem kann offensichtlich elektromagnetische Strahlung vom Menschen gefühlt werden. Die Schwellen hierfür sind individuell sehr unterschiedlich. Es gibt offensichtlich Personen, die sehr empfindlich auf Wechselfelder reagieren, d. h. die bei sehr geringen Leistungen noch elektromagnetische Felder erkennen. Solche Personen werden als ,elektrosensitiv“ bezeichnet (Leitgeb 1998). Von diesem Phänomen der „Hypersensitivität" ist das Phänomen der „Elektrosensibilität“ abzugrenzen. Dabei handelt es sich um Personen, die angeben, unter dem Einfluss hochfrequenter elektromagnetischer Strahlen unter zum Teil schweren gesundheitlichen Beeinträchtigungen zu leiden. Die für die Beschwerden als Ursache angesehene elektromagnetische Strahlung ist dabei meist in einem Dosisbereich weit unterhalb der behördlicherseits vorgegebenen Grenzwerte. Das Krankheitsbild hat, was die Symptome betrifft, viele Gemeinsamkeiten mit dem sogenannten Multiplen Chemikalienempfindlichkeitssyndrom (MCS). ${ }^{3}$ Auch bei Letzterem sind die als ursächlich angesehenen Expositionskonzentrationen potenziell toxischer Substanzen weit unterhalb derer, die bei der Normalbevölkerung gesundheitliche Beeinträchtigungen bewirken. Oft sind die Betroffenen schwerst krank und stehen unter einem enormen Leidensdruck. Nicht selten bezeichnen sich Personen sowohl als MCS-krank als auch als elektrosensibel. 


\section{Eine Fallbeschreibung}

Als Beispiel für das Phänomen der Elektrosensibilität sei der Fall einer Ärztin berichtet, die selbst Betroffene ist ${ }^{4}$. Diese Ärztin schildert ihren Leidensweg wie folgt:

Anfang September 2004 traten zunächst nur kurzzeitige (wenige Minuten andauernde) "Anfälle“ mit Unwohlsein, Angstgefühl, Schwindel, Konzentrationsstörung und dem Gefühl, die Umgebung, ,wie durch einen Nebel“" zu sehen, auf. Erstmals kam es zu nächtlichem Aufwachen mit dem oben beschriebenen Beschwerdebild. Auch wurde ein erhöhter Blutdruck gemessen (150/90 $\mathrm{mmHg}$ ), der früher nicht bekannt war. In den folgenden Wochen kam Kollapsneigung, Druckgefühl über dem Brustkorb, Schmerzen vom Rücken in die linke Brustseite ausstrahlend und Herzrasen hinzu. Der Blutdruck nahm weiter zu (bis 180/110 mmHg). Zusätzlich traten in der Folgezeit Übelkeit, Durchfälle und Muskelzittern wie bei Schüttelfrost auf. Alle in diesem Kontext denkbaren und bei der Patientin durchgeführten internistischen Untersuchungen waren ohne pathologischen Befund. Am 3.10.04 kam es zur Notfallaufnahme im örtlichen Krankenhaus. Es wurde der Verdacht auf ein Phäochromocytom geäußert. Während des stationären Aufenthalts zur Abklärung dieses Verdachts besserten sich die Symptome, traten aber nach Rückkehr in das eigene Haus prompt wieder auf. Ende November waren die Beschwerden so stark, dass innerhalb mehrerer Nächte hintereinander lediglich für zwei bis drei Stunden geschlafen werden konnte. Meist war dies nur in einem der Kinderzimmer möglich, das in einem anderen Stockwerk des Hauses lag. Bei längerer Abwesenheit von zu Hause kam es dagegen immer zu deutlicher Besserung. Anfang Dezember begannen jedoch die Schlafstörungen immer massivere Ausmaße anzunehmen; ,(...) diese veranlassten mich zum Aufsuchen der Neurologin und des Psychotherapeuten. Da ich nicht an eine Depression als Ursache der Beschwerden glaubte, scheute ich mich davor, mit (einer antidepressiven) Medikation zu beginnen. Im Gespräch mit dem Psychotherapeuten wurde mir der psychosomatische Ansatz im Verständnis des Hochdrucks dargelegt. Mögliche Ursachen aus psychischer Sicht für das beschriebene Krankheitsbild aus meiner familiären und beruflichen Situation wurden beleuchtet und zumindest eine Mitverursachung nicht ausgeschlossen. Ich begann über eine psychotherapeutische Behandlung nachzudenken."

Ende Januar 2005 erfährt die Betroffene, eher zufällig, von den Strahlenexpositionen, die von DECT-Telefonen ausgehen sollen. Vom Ehemann daraufhin veranlasste Messungen am Schlafplatz ergaben $400 \mu \mathrm{W} / \mathrm{m}^{2}$, nach Ausschalten des Basisgeräts 10 $\mu \mathrm{W} / \mathrm{m}^{2}$. Das DECT-Telefon war im Hause der Betroffenen seit Sommer 2003 in Betrieb. Die Betroffene dazu: „Ich möchte an dieser Stelle betonen, dass ich vor meiner eigenen Krankheitsgeschichte es für eher unwahrscheinlich hielt, dass überhaupt eine gesundheitliche Gefährdung von Hochfrequenzstrahlung - in Form von Handys und Sendemasten - ausgehen kann. Dass Schnurlostelefone nach dem DECT-Standard auf der gleichen Technik beruhen, wusste ich nicht (hatte mir auch nie besonders Gedanken um derartige Dinge gemacht). Als mein Mann mir vorschlug, in dieser Hinsicht unseren Schlafplatz untersuchen zu lassen, habe ich mir nicht viel Hoffnung gemacht, dass dies eine Lösung meines gesundheitlichen Problems bringen würde, denn ich hatte schon so viele Ansätze verfolgt, die alle keine Besserung gebracht hatten. Noch zu dem Zeitpunkt war meine Einstellung: ,Schaden kann es ja nicht, man darf nichts unversucht lassen.' Ich war letztendlich auch sehr verzweifelt!“

Als sich die Symptomatik nach Umstellung der Basisstation des DECT-Telefons besserte, entschließt sich die Familie statt der DECT-Anlage wieder ein „Schnurtelefon“ zu installieren. „Nach einer Woche sistieren die Durchfälle. Zwei Wochen nach der Umstellung schlafe ich erstmals nachts mit nur kurzen Unterbrechungen. Der Blutdruck lässt sich besser kontrollieren, nach $3 \mathrm{Wo-}$ chen reduziere ich erstmals die antihypertensive Dosis. Im März schlafe ich erstmals ohne nächtliches Aufwachen. Seitdem bin ich beschwerdefrei. Ich messe mehrmals wöchentlich den Blutdruck, der nur selten Werte um 140/80 erreicht.“

Naturheilkundlich ausgerichtete Ärzte, vor allem in Süddeutschland, sammeln und publizieren seit einigen Jahren ähnliche Fälle elektrosensibler Patienten mit dem Ziel, Behörden und Industrie zu bewegen, Maßnahmen zur Reduktion der elektromagnetischen Felder zu 
veranlassen. Trotzdem ist nach wie vor umstritten, ob das Phänomen „Elektrosensibilität“ eine reale somatische Grundlage hat. Im Rahmen des DMF wurden deshalb vier Forschungsprojekte vergeben, die sich mit dieser Frage beschäftigten.

\section{Studien zur Elektrosensibilität}

In der ersten Studie wurden Probanden, die sich selbst als elektrosensibel bezeichneten, und eine nach Alter und Geschlecht entsprechende Kontrollgruppe untersucht (Frick et al. 2006). „Geblindet ${ }^{465}$ wurden den Probanden am Kopf Magnetimpulse mit steigender Intensität appliziert. Dabei konnten die selbsternannten Elektrosensiblen schlechter zwischen realen und Scheinimpulsen unterscheiden als die Kontrollpersonen.

Eine zweite Studie untersuchte den Einfluss einer mobilen Mobilfunk-Sendestation auf das Schlafverhalten (Danker-Hopfe et al. 2008). Sie wurde bundesweit an zehn Standorten in fünf Bundesländern durchgeführt, die sich durch das Fehlen von Mobilfunksignalen sowie geringe andere Hochfrequenzfelder auszeichneten. Der zur nächtlichen Exposition verwendete mobile Sendemast sendete unmodulierte sowie pulsmodulierte Signale nach GSM 900- und GSM 1800-Standard. ${ }^{6}$ Die Exposition erfolge nur in den Expositionsnächten, tagsüber sowie während der Scheinexposition wurde kein Signal abgestrahlt. Die Exposition erfolgte verblindet, das verwendete Testsignal wurde von Mobiltelefonen nicht erkannt. Es ergaben sich keine relevanten Unterschiede bezüglich des Schlafs der Probanden zwischen den Nächten mit Exposition und Scheinexposition.

In der dritten Studie wurde die Schlafqualität subjektiv elektrosensibler Personen untersucht, die unter besonders massiven Schlafstörungen litten und verfestigte Überzeugungen über einen Zusammenhang mit hochfrequenten Immissionen hatten (Leitgeb 2007). In den Wohnungen der Probanden wurde der Schlafplatz durch einen mobilen "Baldachin" aus HF-schirmendem Material abgeschirmt (,verum“). Der Schirmfaktor betrug etwa $20 \mathrm{~dB}$. Zusätzlich wurde ein äuBerlich gleicher nicht schirmender Stoff ver- wendet (,sham“). Unter Kontrollbedingungen war kein Baldachin vorhanden. Es wurden je drei Nächte unter den jeweiligen Bedingungen im randomisierten Doppelblind-Design untersucht. Die Untersuchungen ergaben keinen Hinweis auf gesundheitsschädigende Einflüsse der hochfrequenten elektromagnetischen Immissionen auf den Schlaf, insbesondere auch nicht durch jene des Mobilfunks. Nach Einschätzung des Autors zeigt die Studie jedoch, dass ,subtile Auswirkungen hochfrequenter Immissionen auf das Einschlafverhalten möglich sind. Dieses Ergebnis ist besonders in Hinblick auf die Diskussion über mögliche Wechselwirkungsmechanismen schwacher (nichtthermischer) hochfrequenter elektromagnetischer Felder von Bedeutung und sollte daher durch weitere Untersuchungen abgesichert werden“" (Leitgeb 2007, S. 109).

In der vierten noch nicht mit einem $\mathrm{Ab}$ schlussbericht vorliegenden Studie sollte anhand objektiver medizinischer Tests geklärt werden, ob Allergien und eine besonders hohe Belastung mit bzw. eine erhöhte Empfindlichkeit gegenüber Schwermetallen und Chemikalien bedeutsam für das Auftreten von Elektrosensibilität sind, und wie sich dieser Zusammenhang gegebenenfalls auf Art und Stärke der gesundheitlichen Beeinträchtigungen der Betroffenen auswirkt. Bei dieser Studie ging es unter anderem um den oben erwähnten $\mathrm{Zu}$ sammenhang von Elektrosensibilität und MCS (Dahmen 2005). Es wurden keine signifikanten Unterschiede in den untersuchten somatischmedizinischen Parametern zwischen selbstdefinierten Elektrosensiblen und Kontrollpersonen gefunden. Die Elektrosensiblen zeigten allerdings häufiger somatoforme autonome Funktionsstörungen (38 Prozent).

Trotz des nicht unerheblichen Aufwands hat das DMF mit diesen Untersuchungen wenig dazu beigetragen, die Beurteilung eines Falles wie des oben geschilderten zu erleichtern. Lediglich die Studie von Leitgeb (2007) richtet das Augenmerk direkt auf diese Problematik. Die kleine Zahl von Probanden und die ausschließliche Fokussierung auf die Beeinträchtigung des Schlafes erlauben keine definitive Aussage zur realen Existenz des Phänomens „Elektrosensibilität“, zumal der Autor selbst die Möglichkeit subtiler Effekte erwägt. Die Diagnose einer somatoformen Funktionsstö- 
rung wie bei der Untersuchung von Dahmen führt ebenfalls nicht weiter, da es sich dabei definitionsgemäß um ein psychiatrisches Krankheitsbild handelt, bei dem die betroffenen Personen angeben, unter körperlichen Symptomen zu leiden, für die keine körperliche Ursache gefunden werden kann (Dahmen 2005).

Fälle, wie der oben dargestellte der betroffenen Ärztin, stellen die naturwissenschaftliche Medizin vor eine kaum bewältigbare Herausforderung. Die meisten Symptome solcher Patienten sind funktioneller Art und somit kaum unabhängig von ihrer subjektiven Einschätzung objektivierbar. Sie können die vielfältigsten somatischen oder psychischen Ursachen haben, die durch eine sorgfältige medizinische Untersuchung ausgeschlossen werden müssen. Auch wenn sie nicht im Kontext hochfrequenter elektromagentischer Felder geschildert werden, kann eine konkrete, behebbare Ursache häufig nicht gesichert werden. Die Patienten werden dann eben mit der ,Ausschlussdiagnose" somatoforme Störung abgespeist und damit quasi „psychiatrisiert“.

Soweit es sich um physikalisch messbare Symptome (wie z. B. die Blutdruckerhöhung bei der obigen Patientin) handelt, wären prinzipiell doppelt-blind ${ }^{7}$ angelegte Karenz-Expositionsversuche denkbar. In gewisser Weise hat die geschilderte Patientin quasi einen Selbstversuch durchgeführt, in dem sie das DECTTelefon aus ihrer Umgebung verbannte. Aber könnte das Ergebnis nicht einem PlaceboEffekt geschuldet sein?

Es sind deshalb mehr sorgfältig kontrollierte wissenschaftliche Untersuchungen an betroffenen Einzelpersonen erforderlich. Dem Verfasser sind dabei durchaus die methodischen Probleme bewusst, die sich aus den unterschiedlichen $\mathrm{zu}$ untersuchenden Expositionsbedingungen, der Vielfalt der zu beobachtenden Endpunkte und den möglicherweise komplexen (nicht linearen?) Wirkungsbeziehungen und Latenzzeiten ergeben. Aber abgesehen von dem wissenschaftlichen Interesse an Erkenntnis, haben klinisch tätige Ärzte das Problem, dass es sich bei den Betroffenen um schwer leidende Menschen handelt, denen therapeutisch geholfen werden muss.

\section{Anmerkungen}

1) Der Autor ist stellvertretender Vorsitzender des Ausschusses für Gesundheit und Umwelt der Bundesärztekammer mit Sitz in Frankfurt a. M.

2) Eine umfangreiche Literaturzusammenstellung hierzu kann z. B. der Arbeit von Hecht (2008) entnommen werden.

3) MSC steht für „multiple chemical sensitivity“.

4) Der Fall wurde einer von Frau Dr. med. Cornelia Waldmann-Selsam dem Bundesamt für Strahlenschutz zur Verfügung gestellten Dokumentation entnommen.

5) D. h. die Versuchspersonen wussten nicht, ob sie Magnetpulsen ausgesetzt waren oder nicht.

6) GSM = Global System for Mobile Communication. Dieser technische Standard gilt in fast allen Ländern Europas. GSM900 mit einer Sendefrequenz von ca. $900 \mathrm{MHz}$ wird im D-Netz, GSM 1800 mit einer Sendefrequenz von ca. $1800 \mathrm{MHz}$ wird von E-plus verwandt.

7) Weder die untersuchte Person noch der Untersucher wissen über die im Versuch aktuelle Expositionssituation Bescheid.

\section{Literatur}

Dahmen, N., 2005: Untersuchung elektrosensibler Personen im Hinblick auf Begleitfaktoren bzw. -erkrankungen, wie z. B. Allergien und erhöhte Belastung mit bzw. Empfindlichkeit gegenüber Schwermetallen und Chemikalien - Zwischenbericht an das BfS; http://www.emf-forschungspro gramm.de/forschung/biologie/biologie_verg/bio_11 5.html/bio_115_ZwB_01.pdf (download 15.9.08)

Danker-Hopfe, H.; Dorn, H.; Sauter, C. et al., 2008: Untersuchung der Schlafqualität bei elektrosensiblen Anwohnern von Mobilfunk-Basisstationen unter häuslichen Bedingungen. Abschlussbericht an das BFS; http://www.emf-forschungsprogramm.de/for schung/biologie/biologie_abges/bio_095_AB.pdf (download 15.9.08)

Frick, U.; Hauser, S.; Landgrebe, M. et al., 2006: Untersuchung des Phänomens „Elektrosensibilität“ mittels einer epidemiologischen Studie an ,elektrosensiblen“ Patienten einschließlich der Erfassung klinischer Parameter. Endbericht an das Bundesamt für Strahlenschutz; http://www.emf-forschungspro gramm.de/forschung/biologie/biologie_abges/bio_0 15_AB.pdf (download 6.9.08)

Hecht, K., 2008: Wissenschaftliche Tatsachen aus der internationalen Forschung zur biologischen Wirkung von Hochfrequenzstrahlen auf den Menschen und zum Radiofrequenz-(Mikrowellen-)Syndrom 
Leitgeb, N., 1998: Electromagnetic Hypersensitivity. Paper presented at the International Workshop on Electromagnetic Fields and Non-specific Health Symptoms. Graz, Österreich

Leitgeb, N., 2007: Untersuchung der Schlafqualität bei elektrosensiblen Anwohnern von MobilfunkBasisstationen unter häuslichen Bedingungen. Abschlussbericht an das BfS; http://www.emfforschungsprogramm.de/forschung/biologie/biolo gie_abges/bio_096_AB.pdf (download 6.9.08)

Nagelschmidt, K.F., 1909: Über Diathermie (Transthermie, Thermopenetration). In: Münchener Medizinische Wochenschrift 56 (1909), S. 2575

Schliephake, E., 1932: Arbeitsgebiete auf dem Kurzwellengebiet. In: Deutsche Medizinische Wochenschrift 32 (1932), S. 1235

Schwan, H.P., 1957: Electrical Properties of Tissues and Cells. In: Advances in Biological and Medical Physics 5 (1957), S. 147-209

Schwan, H.P.; Piersol, G.M., 1954: The Absorption of Electromagnetic Energy in Body Tissues. Pt. I. In: American Journal of Physics in Medicine 33/6 (1954), S. 371-404

Schwan, H.P.; Piersol, G.M., 1955: The Absorption of Electromagnetic Energy in Body Tissues. Pt. II. In: American Journal of Physics in Medicine 34 (1955), S. 425-448

SSK - Strahlenschutzkommission, 2006: Mobilfunk und Kinder - Stellungnahme der Strahlenschutzkommission und wissenschaftliche Begründung; http:/www.ssk.de/werke/volltext/2006/ssk0619.pdf (download 13.9.08)

von Mühlendahl, K.F.; Otto, M., 2008: Umweltmedizin in der Pädiatrie. Warum gibt es hier Besonderheiten? Wie geht man mit ihnen um? In: Umweltmedizin in Forschung und Praxis 13, S. 145-153

\section{Kontakt}

Prof. Dr .med. Dr. phil. nat. Andreas D. Kappos

Bundesärztekammer

Im Geeren 36, 60433 Frankfurt

Tel.: +49 (0) 69 / 95775573

E-Mail: adkappos@t-online.de

\section{Mobilfunk-Infrastruktur und Schutz der Bevölkerung aus Sicht von Regierung und Politik}

\author{
von Axel Böttger, Bundesministerium für \\ Umwelt, Naturschutz und Reaktorsicherheit ${ }^{1}$
}

Mit der Einführung des digitalen Mobilfunks in Deutschland wurde es für breite Bevölkerungskreise möglich, zu jeder Zeit und an jedem Ort telefonieren zu können. Der Beginn des „Mobilfunkzeitalters“ wurde aber auch von einem Unbehagen gegenüber dieser neuen Technik begleitet, gesundheitliche Auswirkungen wurden befürchtet. Frühzeitig war deshalb die Bundesregierung initiativ geworden und schuf in Folge weltweit eine der ersten gesetzlichen Regelungen zum Schutz der Bevölkerung. Verbindliche Grenzwerte wurden u. a. für Mobilfunk-Basisstationen eingeführt. Trotz einer allgemeinen Beruhigung der Debatte bestand das Unbehagen in der Bevölkerung teilweise fort und wurde durch die Einführung der neuen UMTS-Technik sogar wieder verstärkt. Daraufhin versuchte die Bundesregierung die Kenntnislücken, die von der Strahlenschutzkommission identifiziert worden waren, mithilfe des „Deutschen MobilfunkForschungsprogramms" (DMF) zu schließen. Parallel sagten die Mobilfunknetzbetreiber gegenüber der Bundesregierung in einer Selbstverpflichtung eine Reihe von Maßnahmen insbesondere zur Kommunikation und zur Information der Bevölkerung zu und führten diese auch durch. Im Juni 2008 wurde das DMF abgeschlossen; ein Ergebnis dieses Programms war, dass sowohl das Bundesamt für Strahlenschutz als auch die Strahlenschutzkommission der Bundesregierung empfahlen, an den geltenden Grenzwerten festzuhalten.

\section{Erste gesetzliche Regelung}

Mit der bundesweiten Einführung des digitalen Mobilfunks zu Beginn der 1990er Jahre war in der Bevölkerung insgesamt und in der Industrie eine deutliche Begeisterung für die neue Technik zu spüren. Gleichzeitig kam jedoch in bestimmten Bevölkerungsgruppen Skepsis gegenüber der für sie nicht einschätzbaren neuen Technik auf. Diese Befürchtungen vor möglichen gesundheitlichen Auswirkungen wurden 